\title{
Des particules qui se la coulent douce...
}

Bloen Metzger (bloen.metzger@polytech.univ-mrs.fr) et Élisabeth Guazzelli

IUSTI, UMR CNRS 6595, Technopôle de Château-Gombert, 13453 Marseille Cedex 13

Formation d'un tore,

déstabilisation en gouttelettes,

puis cascade auto-similaire :

la sédimentation d'un nuage

de particules dans un fluide

visqueux est spectaculaire.

Comment un système

conceptuellement aussi simple

peut-il développer une telle

richesse de comportements ?

La longue portée des

interactions hydrodynamiques

entre particules

et le comportement collectif

qu'elles entraînent (du type

problème à N-corps) sont

les composantes essentielles

pour comprendre cette

dynamique.
Lorsque de petites particules sédimentent (tombent sous l'effet de la gravité) à faible vitesse dans un fluide initialement au repos, elles génèrent des perturbations d'écoulement dont la décroissance spatiale est très lente. Les particules s'influencent mutuellement via le fluide. Chaque particule voit ainsi sa trajectoire modifiée par rapport à celle qu'elle aurait si elle tombait de façon isolée.

Ces interactions hydrodynamiques, propres aux écoulements de Stokes (encadré, p. 9), sont à l'origine de phénomènes complexes d'un grand intérêt. Cette situation préfigure de nombreux phénomènes naturels comme le comportement de globules rouges, la sédimentation d'alluvions ou, pour citer une expérience quotidienne, la chute des grains de marc dans votre tasse de café. Ce système donne surtout une occasion quasiment unique en physique classique d'étudier expérimentalement le problème à $\mathrm{N}$-corps ${ }^{(1)}$. En effet, à l'instar de l'interaction gravitationnelle qui régit le mouvement des planètes, les interactions hydrodynamiques entre particules sont de type longue portée.

Nous commencerons par décrire la dynamique de sédimentation d'une, de deux, puis de trois particules. Au passage de deux à trois particules, on aborde le problème à N-corps ; apparaissent alors certains comportements chaotiques. La deuxième partie de l'article est consacrée à la sédimentation d'un " nuage de particules ». Des films sont disponibles sur le lien de la référence [1].

\section{$1,2,3$ particules}

Le problème de la chute d'une particule sphérique dans un fluide visqueux sous l'effet de la gravité a été complètement résolu par Stokes en 1851 [2]. La perturbation d'écoulement induite au sein du fluide par la chute de la sphère possède une propriété remarquable : son amplitude décroît très lentement, comme l'inverse de la distance au centre de la sphère (encadré, p. 9). On verra que cette propriété est essentielle pour comprendre la dynamique d'évolution d'un ensemble de particules.

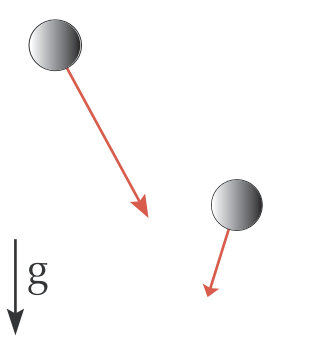

(a)

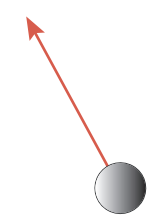

$\uparrow g$

(b)
1. Deux sphères identiques tombent lentement dans un fluide visqueux sous l'effet de leur propre poids : la linéarité des équations de Stokes permet de montrer que les vitesses des deux sphères sont nécessairement les mêmes.

a) Supposons que les deux sphères ont des vitesses différentes: la sphère située à l'arrière (par rapport au sens du mouvement) rattrape la première sphère et les deux sphères se rapprochent. b) Inversons le sens de la pesanteur $\mathbf{g}$. En utilisant la propriété de linéarité, l'inversion du sens des forces entraîne l'inversion du sens des vitesses. Cette fois, la sphère située à l'avant se déplace plus rapidement et les deux sphères s'éloignent l'une de l'autre.

Les deux situations sont pourtant formellement identiques, la dynamique doit donc nécessairement être la même. Les vitesses des deux sphères sont donc identiques.

Imaginons maintenant la sédimentation d'un doublet de particules identiques. Le mouvement du fluide induit par le déplacement de chacune des sphères influence la trajectoire de l'autre. La vitesse de chaque sphère peut ainsi être évaluée en ajoutant la vitesse propre de sédimentation d'une sphère isolée à celle induite dans le fluide par le mouvement de l'autre. La vitesse du doublet de particules a donc un module supérieur à la vitesse de sédimentation d'une particule isolée (fig. 2 - a et b). La linéarité des équations de Stokes permet de montrer que les vitesses résultantes des deux sphères sont nécessairement identiques (fig. 1). Les deux particules sédimentent donc sans mouvement relatif l'une par rapport à l'autre.

La situation est très différente pour trois particules [3]. Certaines configurations initiales très particulières produisent un mouvement périodique (fig. 2c) : lorsque, par exemple, les trois particules sont initialement positionnées aux sommets d'un triangle isocèle horizontal. Dans 


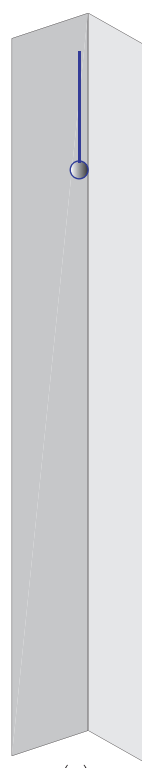

(a)

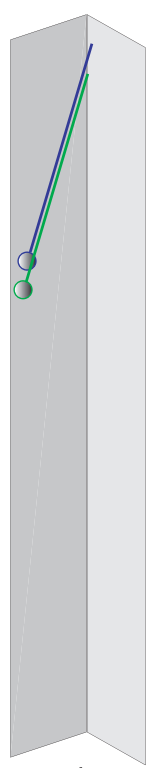

(b)

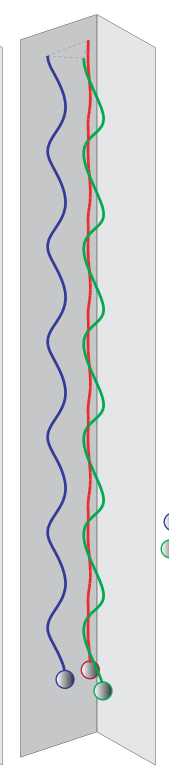

(c)

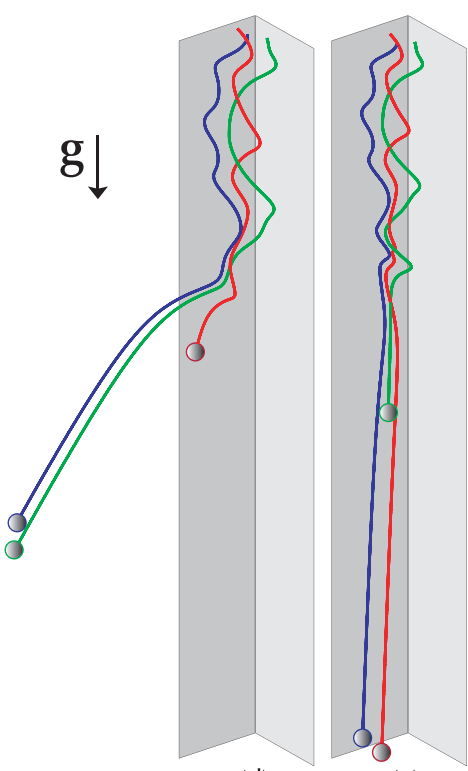

(d) (e)
2. Simulations numériques : sédimentation d'une (a), de deux (b) ou de trois (c, d, e) particules dans un fluide visqueux.

c) Initialement positionnées aux sommets d'un triangle isocèle horizontal, les particules suivent un mouvement périodique.

d) Initialement distribuées aléatoirement, les particules passent par une phase d'interaction à trois, puis un doublet (particules bleue et verte) «s'échappe » et laisse la particule rouge isolée.

e) Même configuration initiale que pour d, sauf pour la particule bleue dont la position initiale a été légèrement modifiée. Si l'évolution aux temps courts est similaire, l'évolution aux temps longs, par contre, est très différente d'une configuration à l'autre. Cette fois, le doublet rouge et bleu s'échappe et laisse la particule verte isolée. Cette extrême sensibilité aux conditions initiales est caractéristique des systèmes chaotiques. les autres cas, la dynamique d'évolution est chaotique (fig. $2-\mathrm{d}$ et e). On distingue une phase d'interaction à trois particules, pendant laquelle la position relative des particules change totalement. Ensuite, un doublet "s'échappe » et laisse en arrière la troisième particule. Le doublet sédimente plus rapidement que la particule isolée, car chacune des particules du doublet est entraînée par la perturbation d'écoulement générée par l'autre particule du doublet. La nature chaotique de la configuration à trois particules est bien illustrée dans la figure 2 (d et e) par la sensibilité du système aux conditions initiales.

Les équations de Stokes qui déterminent le mouvement de ces particules sont linéaires et instantanées. Elles sont par conséquent réversibles en temps. Comment se fait-il alors que les particules présentent une dynamique d'évolution si complexe ? Pourquoi une telle sensibilité aux conditions initiales ? Cette dynamique chaotique apparaît dès que le nombre de particules est supérieur à deux. Elle a pour origine le couplage entre l'écoulement du fluide et la distribution spatiale des particules. À un instant donné, la distribution spatiale des particules détermine complètement l'écoulement, mais l'écoulement produit entraîne les particules et donc modifie aussitôt cette distribution. Cette perpétuelle rétroaction entre écoulement et microstructure donne lieu à une dynamique temporelle fortement non linéaire, qui est à l'origine du comportement chaotique observé.

\section{Chute d'une petite particule sphérique dans un fluide visqueux sous l'effet de la gravité.}

Lorsqu'une petite particule de rayon a tombe dans un fluide visqueux de viscosité $\mu$, la force de traînée $\mathbf{T}=-6 \pi \mu \mathrm{U} \mathbf{U}$ exercée par le fluide sur la particule compense exactement la force de gravité $\mathbf{F}$, de sorte que la vitesse de sédimentation de la particule $\mathbf{U}=\mathbf{F} / 6 \pi \mu$ a est constante. L'écoulement $\mathbf{u}$ induit dans le fluide est solution des équations de Stokes (1), où $p$ est le champ de pression et $\delta$ la fonction de Dirac qui décrit la distribution du champ de forces au sein du fluide, si on assimile la particule à une masse ponctuelle. Ces équations, valables si l'écoulement est suffisamment lent et/ou la viscosité du fluide suffisamment grande et/ou la taille de la particule suffisamment petite, sont linéaires ; elles sont donc aussi réversibles (pour plus de détails, consultez la référence [1]). La solution analytique de cet écoulement est connue (2) ; elle correspond à la solution de Green des équations de Stokes.

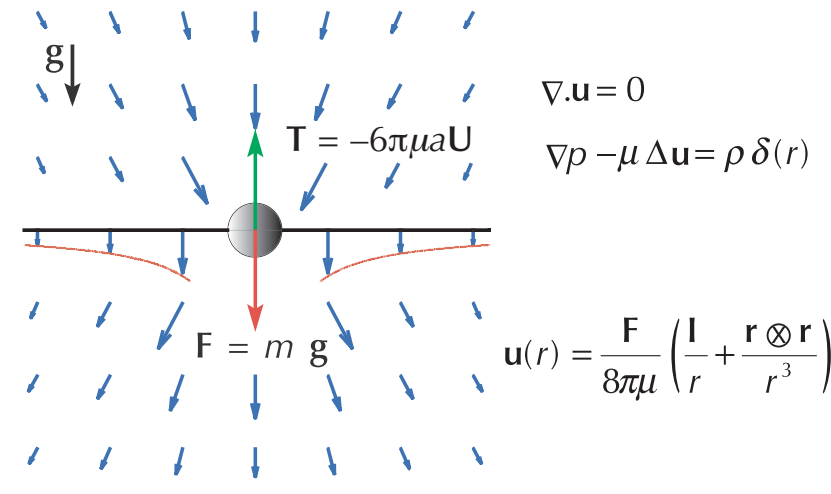

Sur la figure, les flèches bleues représentent le champ de vitesse induit dans le fluide par la chute de la particule, et la courbe rouge indique sa propriété la plus remarquable, qui est la lente décroissance (en 1/r) de son amplitude. 


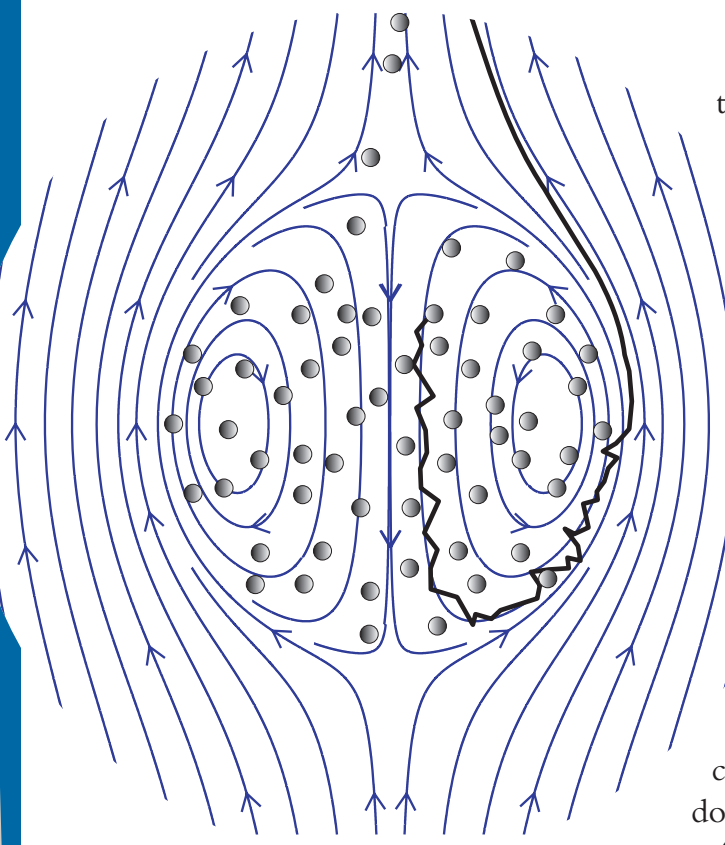

3. Simulation numérique de l'écoulement produit (dans le référentiel du nuage) par un nuage de particules. Le mouvement général est celui d'une recirculation toroïdale d'axe vertical. Les fluctuations dues au caractère discret et aléatoire de la distribution des particules à l'intérieur du nuage conduisent certaines particules à traverser l'interface entre le nuage et le fluide externe. Ces particules, entraînées par le fluide externe, forment un fin filet à l'arrière du nuage.

$>>>$

\section{Nuage de particules}

Nous allons maintenant examiner le mouvement d'un grand nombre de particules soumises à la gravité. Les particules sont initialement distribuées aléatoirement à l'intérieur d'un volume prescrit du fluide, de sorte qu'elles forment ce que l'on appellera dans la suite un "nuage de particules ».

Le phénomène le plus surprenant, et certainement le plus spectaculaire, est la dynamique collective des particules pendant leur chute (fig. 3). Le mouvement général apparent est celui d'une recirculation toroïdale d'axe vertical. Remarquez que ce mouvement collectif résulte uniquement des interactions hydrodynamiques entre particules.

Un modèle simple pour décrire ces interactions est de considérer que chaque particule produit dans le fluide ambiant une perturbation d'écoulement équivalente à celle que produirait à sa place une particule ponctuelle(2) (encadré, p. 9). Cette approximation est naturelle en milieu dilué, puisque la distance entre les particules est grande comparée à leur taille typique ; les particules ne ressentent alors que l'ordre dominant des perturbations d'écoulement créées par leurs voisines (en $1 / r$ ).

La linéarité des équations de Stokes peut nous aider à comprendre la dynamique collective des particules. Chacune des particules à l'intérieur du nuage est advectée par la somme des perturbations d'écoulement induites par la chute de toutes les autres particules du nuage. Comme deux particules voisines voient quasiment la même distribution de particules autour d'elles, elles ont des vitesses presque identiques et donc des trajectoires fortement similaires ; au lieu de se disperser rapidement dans le fluide ambiant, les particules du nuage forment une entité "cohésive " qui perdure pendant un temps très long.

Ce mouvement de recirculation toroïdal est aussi observé lorsqu'une goutte de fluide pur sédimente dans un fluide plus léger [4]. La suspension peut être assimilée à un fluide effectif plus dense que le fluide ambiant, mais cette analogie a des limites ; les suspensions possèdent des caractéristiques intrinsèques qui ne peuvent être traduites dans un modèle de type milieu effectif.
Le mécanisme de perte de particules illustre bien ces différences. Certaines particules s'échappent spontanément du nuage (fig. 3). Le caractère multiple des interactions et le réarrangement permanent des particules au sein du nuage génèrent d'importantes fluctuations (comme l'illustre schématiquement la ligne brisée de la figure 3). Certaines particules traversent spontanément l'interface entre le nuage et le fluide externe, et sont alors entraînées par ce dernier. Malgré la nature réversible des équations de Stokes, on observe un mécanisme qui génère un transport diffusif irréversible des particules vers l'extérieur du nuage.

Les particules qui "s'échappent " viennent de la couche externe du mouvement toroïdal de recirculation du nuage ; leur départ crée un déficit de particules au niveau de son axe vertical de symétrie (fig. 4). Le nuage initialement sphérique évolue donc lentement vers un tore.

Le tore continue de perdre des particules et son rapport d'aspect (rapport de sa dimension horizontale sur sa dimension verticale) augmente. Au-delà d'un rapport d'aspect critique, la topologie de l'écoulement change brutalement : le fluide externe passe alors au centre de l'anneau formé par le nuage au lieu de le contourner (fig. 4). À cet instant précis, le tore se fragmente en plusieurs gouttelettes. Chaque gouttelette forme un tore plus petit qui se déstabilise à son tour, formant ainsi une cascade de gouttelettes de particules (fig. 5).

Cette déstabilisation nous donne l'occasion de revenir sur la notion de rétroaction entre microstructure et écoulement. Juste avant la déstabilisation, la distribution spatiale des particules est telle que l'écoulement du fluide externe contourne le nuage de particules : le tore est stable (fig. 4). Au-delà du rapport d'aspect critique, i.e. pour une
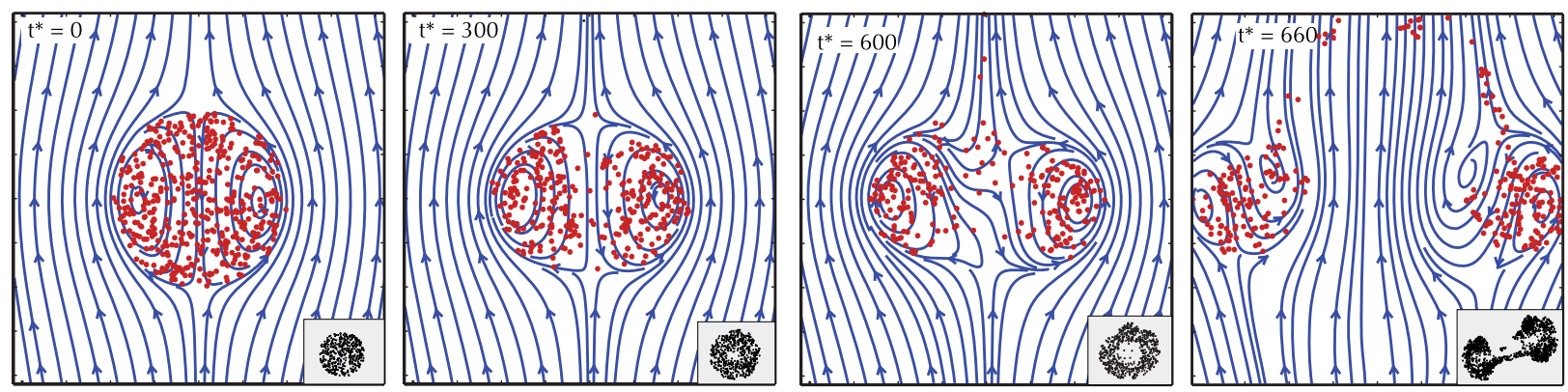

4. Simulation numérique de l'écoulement (dans le référentiel du nuage) généré par un nuage comportant initialement 3000 particules. Le nuage, initialement sphérique $\left(t^{*}=0\right)$, évolue progressivement vers un tore $\left(t^{*}=300\right)$. Lorsque le fluide externe passe au centre du nuage $\left(t^{*}>600\right)$, il y a déstabilisation en deux gouttelettes similaires au nuage initial. Les inserts représentent les vues de dessous correspondantes. Le temps est adimensionné par le temps mis par le nuage pour parcourir son rayon : $t^{*}=t\left(R / V_{n}\right)$, où $R$ et $V_{n}$ sont respectivement le rayon et la vitesse de sédimentation du nuage à $t^{*}=0$. 
microstructure légèrement différente, le fluide externe passe au centre de l'anneau formé par les particules et le sens de recirculation est alors nécessairement inversé. Ce changement topologique de l'écoulement rend le tore instable : il se brise en gouttelettes, et la distribution spatiale des particules est ainsi totalement modifiée.

\section{Conclusion}

Malgré sa simplicité conceptuelle, la sédimentation d'un nuage de particules dans un fluide visqueux présente une grande richesse de comportements. Dès que le nombre de particules est supérieur à deux, le système devient chaotique. Les trajectoires des particules sont tortueuses et extrêmement sensibles aux conditions initiales. On se retrouve dans la situation du problème à trois corps. La sédimentation donne une occasion quasiment unique d'étudier expérimentalement ce problème, considéré comme l'un des plus difficiles des mathématiques. Pour approfondir ce point, le lecteur peut se reporter à la référence [3].

Pour un nombre plus élevé de particules, l'évolution est plus complexe. Le « nuage de particules " évolue d'abord vers un tore, qui se déstabilise ensuite en gouttelettes plus petites. Les équations qui gouvernent le mouvement des particules sont linéaires et donc réversibles en temps. On observe pourtant un certain nombre de phénomènes irréversibles comme la perte de particules, qui sont déterminants dans l'évolution subséquente du nuage. Un modèle numérique très simple, basé sur une approximation de particules ponctuelles est suffisant pour capturer l'évolution du nuage jusque dans ses détails (fig. 5). Cette étude montre à quel point les interactions hydrodynamiques dominent la dynamique de ces systèmes.

Un autre phénomène saisissant est le mouvement général de recirculation toroïdale observé par les particules. Cette dynamique collective,

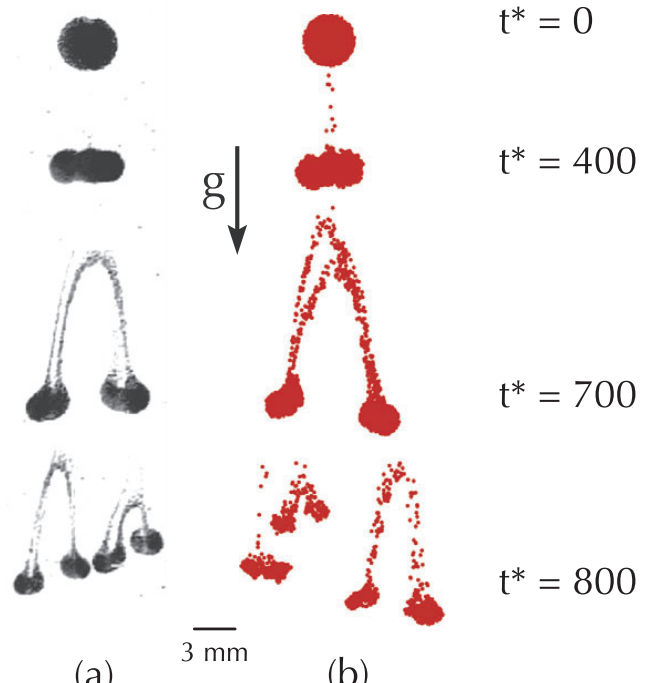

5. Images successives de la chute d'un nuage de particules tombant dans un liquide visqueux.

a) Expérience de laboratoire réalisée à l'aide de particules de verre de diamètre $100 \mu \mathrm{m}$ dans une cuve remplie d'huile silicone 1000 fois plus visqueuse que l'eau. b) Simulation numérique [5]. Des films sont en visualisation et téléchargement libre sur le lien de la référence [1].

\section{Réérérences}

qui naît de la lente décroissance des interactions hydrodynamiques, peut paraître assez singulière. Elle est en fait d'une importance majeure dans un très grand nombre de systèmes moins académiques. On observe, par exemple, des " tourbillons » dans les suspensions de particules sphériques [6] ; des " amas ", qui s'organisent ensuite en colonnes, caractérisent la sédimentation des suspensions de fibres [7]. Ces structures, qui naissent des effets collectifs et qui se développent en général sur des échelles bien supérieures à la taille typique des particules, ont une influence profonde sur la dynamique de ces systèmes. Il est donc particulièrement important de les étudier.

(1) Le problème à $\mathrm{N}$-corps consiste à prédire la dynamique d'un grand nombre de corps ou particules (atomes, électrons...), qui interagissent via des interactions souvent fortes et à longue portée. En physique classique, on le rencontre en astronomie (étude des trajectoires de $\mathrm{N}$ corps célestes en interaction gravitationnelle). On le rencontre aussi en physique des solides et dans d'autres systèmes quantiques.

(2) Cette approximation est équivalente à celle effectuée en astronomie : remplacer deux solides à répartition sphérique de masse par deux masses ponctuelles coïncidant avec leurs centres respectifs.
1 - http://iusti.polytech.univ-mrs.fr / metzger/CloudSedimentation.html

2•É. Guyon, J.P. Hulin et L. Petit, Hydrodynamique physique, Savoirs Actuels, EDP Sciences/CNRS Éditions (1982)

• • I.M. Jánosi, T. Tél, D.E. Wolf, J.A.C. Gallas, "Chaotic particle dynamics in viscous flows: The three-particle Stokeslet problem", Phys. Rev. E 56 (1997) 2858-2868.

4 - J.S. Hadamard, « Mouvement permanent lent d'une sphère liquide et visqueuse dans un liquide visqueux " C. R. Acad. Sci. (Paris) 152 (1911) 1735-1738.

5 • B. Metzger, M.L. Nicolas et É. Guazzelli, "Falling clouds of particles in viscous 283-301.

6 • É. Guazzelli, "Evolution of particlevelocity correlations in sedimentation", Phys. Fluids, 13 (2001) 1537-1540.

7 • B. Metzger, J. E. Butler et É. Guazzelli, "Experimental investigation of the instability of a sedimenting suspension of fibres", J. Fluid Mech., 575 (2007) 307-332. fluids", J. Fluid Mech., 580 (2007) 